\title{
REGULAR COVERINGS OF HOMOLOGY 3-SPHERES BY HOMOLOGY 3-SPHERES
}

\author{
E. LUFT AND D. SJERVE
}

\begin{abstract}
We study 3-manifolds that are homology 3-spheres and which admit nontrivial regular coverings by homology 3-spheres. Our main theorem establishes a relationship between such coverings and the canonical covering of the 3-sphere $S^{3}$ onto the dodecahedral space $D^{3}$. We also give methods for constructing irreducible sufficiently large homology 3-spheres $\widetilde{M}, M$ together with a degree 1 map $h: M \rightarrow D^{3}$ such that $\widetilde{M}$ is the covering space of $M$ induced from the universal covering $S^{3} \rightarrow D^{3}$ by means of the degree 1 map $h: M \rightarrow D^{3}$. Finally, we show that if $p: \widetilde{M} \rightarrow M$ is a nontrivial regular covering and $\widetilde{M}, M$ are homology spheres with $M$ Seifert fibered, then $\widetilde{M}=S^{3}$ and $M=D^{3}$.
\end{abstract}

\section{INTRODUCTION}

The dodecahedral space $D^{3}$ is the only known irreducible 3-manifold with finite (nontrivial) fundamental group, that is also a homology 3-sphere. It is covered by the 3 -sphere. The fundamental group $\pi_{1}\left(D^{3}\right)$ of $D^{3}$ is the binary icosahedral group, denoted by $I^{*}$.

In this paper we investigate those 3-manifolds that are homology 3-spheres and which admit a nontrivial regular covering by a homology 3-sphere. Our main result is the following.

Main Theorem. Let $M, \widetilde{M}$ be homology 3-spheres and $p: \widetilde{M} \rightarrow M$ a nontrivial regular covering. Then the following hold:

(1) The group of covering transformations of $p: \widetilde{M} \rightarrow M$ is the binary icosahedral group $I^{*}$.

(2) The mapping cone $C_{p}$ of $p: \widetilde{M} \rightarrow M$ is homotopy equivalent to the mapping cone $C_{q}$ of the universal covering $q: S^{3} \rightarrow D^{3}$.

(3) There is a map $f: M \rightarrow D^{3}$ with $f_{*}\left(\pi_{1}(M)\right)=\pi_{1}\left(D^{3}\right)$, such that the degree of $f$ is relatively prime to 120 and

$$
p_{*}\left(\pi_{1}(\widetilde{M})\right)=\operatorname{ker}\left(f_{*}: \pi_{1}(M) \rightarrow \pi_{1}\left(D^{3}\right)\right),
$$

that is, the covering $p: \widetilde{M} \rightarrow M$ is the pullback of the covering $q: S^{3} \rightarrow D^{3}$.

Received by the editors May 14, 1987.

1980 Mathematics Subject Classification (1985 Revision). Primary 57M99.

Key words and phrases. Homology 3-spheres, coverings, binary icosahedral group, dodecahedral space, degree 1 maps.

Research partially supported by NSERC grants A3503 and A7218. 
If the homology 3-sphere $M$ is not irreducible it can be decomposed into a connected sum of irreducible homology 3 -spheres, and this will induce a corresponding decomposition of $\widetilde{M}$ and the covering $p: \widetilde{M} \rightarrow M$ (Theorem (3.6)).

If the homology 3-sphere $M$ admits a Seifert fibration and if it also admits a nontrivial regular covering by a homology 3 -sphere $\widetilde{M}$, then necessarily $M=$ $D^{3}$ and $\widetilde{M}=S^{3}$ (Theorem (4.1)).

There is an abundance of irreducible homology 3-spheres that admit nontrivial regular coverings by homology 3 -spheres. In $\S 5$ we construct examples by utilizing the dodecahedral space $D^{3}$. The irreducible homology 3 -spheres in these examples are all sufficiently large. This raises the following question:

Question. Is there an example of an irreducible homology 3-sphere (with infinite fundamental group) that is not sufficiently large or is hyperbolic and that is regularly covered by a homology 3 -sphere?

It is a well-known conjecture that the fundamental group of a compact 3manifold $M$ is residually finite, i.e. there is a sequence $\left\{G_{i}\right\}_{i=1,2, \ldots}$ of subgroups of finite index in $\pi_{1} M$ with $\bigcap_{i} G_{i}=1$. Applying statement 1 of our Main Theorem we obtain that if $M$ is a homology 3-sphere such that $\pi_{1} M$ has a subgroup of finite index which is not a divisor of 120 , then there are infinitely many distinct subgroups of finite index in $\pi_{1} M$ (Corollary 3.2).

\section{Preliminaries}

In this section we collect the background material we need in order to prove our theorems. We will work throughout in the PL category. A PL homeomorphism we simply call an isomorphism. Our reference for 3-manifold concepts is $[\mathrm{He}]$.

By the term surface we will mean a compact, connected 2-manifold. A closed surface $F$ in a 3-manifold $M$ is said to be incompressible if it is not a 2-sphere and if for each 2-cell $B \subset M$ with $B \cap F=\partial B$, there is a 2-cell $B^{\prime} \subset F$ with $\partial B=\partial B^{\prime}$.

A 3-manifold $M$ is said to be irreducible if each 2-sphere in $M$ bounds a 3-cell in $M$. If $p: \widetilde{M} \rightarrow M$ is a covering onto the orientable 3-manifold $M$, then $\widetilde{M}$ is irreducible if and only if $M$ is irreducible [MSY].

A closed orientable connected 3-manifold is sufficiently large if it is irreducible and if it contains a 2 -sided incompressible closed surface.

The following will be used in $\S 5$.

Lemma (2.1). Let $M$ be a 3-manifold and $M_{1}, M_{2}$ submanifolds such that $M=M_{1} \cup M_{2}$ and $M_{1} \cap M_{2}=\partial M_{1} \cap \partial M_{2}=F$ is a component of $\partial M_{1}$ and $\partial M_{2}$. If $M_{1}$ and $M_{2}$ are irreducible and if $F$ is incompressible in $M_{1}$ and $M_{2}$, then $M$ is irreducible. 
There are various descriptions of the dodecahedral space, for example, see [Ro]. We will use the following presentation as a Seifert fibered space. For basic definitions regarding Seifert fibrations of 3-manifolds we refer to [O] or [S].

Let $S^{2}$ be the 2-sphere and let $B_{0}, B_{1}, B_{2}, B_{3} \subset S^{2}$ be four disjoint 2-cells. Then $S_{4}^{2}=\overline{S^{2}-\left(B_{0} \cup B_{1} \cup B_{2} \cup B_{3}\right)}$ is a 2-sphere with 4 holes. Let $S^{1}$ be the 1-sphere. $\partial\left(S_{4}^{2} \times S^{1}\right)$ consists of the 4 tori $\partial B_{0} \times S^{1}, \ldots, \partial B_{3} \times S^{1}$. We give $S_{4}^{2}$ and $S^{1}$ fixed orientations. These define a unique orientation on $S_{4}^{2} \times S^{1}$. Then the dodecahedral space $D^{3}$ is obtained from $S_{4}^{2} \times S^{1}$ by attaching 4 solid tori $B_{0}^{\prime} \times S^{1}, \ldots, B_{3}^{\prime} \times S^{1}$ to the 4 boundary tori of $S_{4}^{2} \times S^{1}$ via isomorphisms $h_{i}: \partial B_{i}^{\prime} \times S^{1} \rightarrow \partial B_{i} \times S^{1}, i=0,1,2,3$, that satisfy

$$
\begin{aligned}
h_{0 *}\left[\partial B_{0}^{\prime}\right] & =\left[\partial B_{0}\right]-\left[S^{1}\right] \quad \text { in } H_{1}\left(\partial B_{0} \times S^{1}\right), \\
h_{i *}\left[\partial B_{i}^{\prime}\right] & =\alpha_{i}\left[\partial B_{i}\right]+\left[S^{1}\right] \quad \text { in } H_{1}\left(\partial B_{i} \times S^{1}\right), 1 \leq i \leq 3,
\end{aligned}
$$

where $\alpha_{i}=5,2,3$, respectively. That is,

$$
D^{3}=\left(S_{4}^{2} \times S^{1}\right) \cup_{h_{0}}\left(B_{0}^{\prime} \times S^{1}\right) \cup_{h_{1}}\left(B_{1}^{\prime} \times S^{1}\right) \cup_{h_{2}}\left(B_{2}^{\prime} \times S^{1}\right) \cup_{h_{3}}\left(B_{3}^{\prime} \times S^{1}\right) .
$$

Thus the dodecahedral space $D^{3}$ is a Seifert fibered space having 3 singular fibers with Seifert invariants $(5,1),(2,1),(3,1)$ determined by the solid tori $B_{1}^{\prime} \times S^{1}, B_{2}^{\prime} \times S^{1}, B_{3}^{\prime} \times S^{1}$, respectively, and with Seifert surface a 2-sphere. The solid torus $B_{0}^{\prime} \times S^{1}$ determines a regular fiber. In the terminology of [S], $D^{3}$ has the description

$$
(0,0 ; \text { ol }-1 ; 5,1 ; 2,1 ; 3,1) .
$$

The fundamental group $\pi_{1}\left(D^{3}\right)$ is the binary icosahedral group $I^{*}$. It has order 120 and its center is a cyclic group of order 2. Each regular fiber $S_{0}^{1} \subset D^{3}$ defines a generator $\left[S_{0}^{1}\right] \in \pi_{1}\left(D^{3}\right)$ of the center. In $\S 5$ we will give a more detailed description of the universal covering $q: S^{3} \rightarrow D^{3}$ of the 3-sphere $S^{3}$ onto the dodecahedral space $D^{3}$.

For any group $G$ let $\varepsilon: Z[G] \rightarrow Z$ denote the augmentation homomorphism of the integral group ring $Z[G]$ and $A[G]=\operatorname{ker} \varepsilon$ the augmentation ideal. If $G$ is a finite group then let $N$ denote the norm element, $N=\sum_{x \in G} x$ in $Z[G]$.

For any integer $r$ the left ideal generated by $r$ and $N$ in $Z[G]$ is denoted by $(r, N)$. If $r$ is relatively prime to the order of $G$ then the ideal $(r, N)$ is a finitely generated projective $Z[G]$-module [ $\left[\mathrm{Sw}_{1}\right.$, Proposition 7.1, p. 570]. Therefore $(r, N)$ determines an element, denoted by $[r, N]$, of the reduced Grothendieck group $\widetilde{K}_{0}(Z[G])$ of finitely generated projective $Z[G]$-modules.

A $(G, m)$-complex is a finite connected $m$-dimensional $C W$ complex $X$ such that $\pi_{1}(X) \cong G$ and the universal covering space $\widetilde{X}$ is $(m-1)$-connected. To any $(G, m)$-complex $X$ there is associated its algebraic $m$-type, that is the triple $T(X)=\left(\pi_{1}(X), \pi_{m}(X), k(X)\right)$ where $k=k(X) \in H^{m+1}\left(G, \pi_{m}(X)\right)$ is the $k$-invariant (see [D, p. 249]). 
An abstract $m$-type is a triple $T=\left(G, \pi_{m}, k\right)$, where $G$ is a group, $\pi_{m}$ is a $Z[G]$-module, and $k \in H^{m+1}\left(G, \pi_{m}\right)$. There are notions of homomorphism and isomorphism for abstract $m$-types (see [D, p. 250] for details).

Theorem (2.2) (see [D]). Two $(G, m)$-complexes $X, Y$ are homotopically equivalent if, and only if, $T(X)$ and $T(Y)$ are isomorphic as abstract $m$-types.

Let $Z_{n}$ denote the ring of integers $\bmod n$ and let $Z_{n}^{*} \subset Z_{n}$ denote its group of units. Now let $X$ be a $(G, m)$-complex, where $G$ is a group of order $n$. Then $H^{m+1}\left(G, \pi_{m}(X)\right) \cong Z_{n}$ and the only $k$-invariants $r$ which can possibly arise from $(G, m)$-complexes with algebraic $m$-type $\left(G, \pi_{m}(X), r\right)$ must be in $Z_{n}^{*} \subset Z_{n}$ (see [D]). By [D, Theorem 2.5], the map $\nu: Z_{n}^{*} \rightarrow \widetilde{K}_{0}(Z[G])$, $\nu(r)=[r, N]$ is a homomorphism.

Theorem (2.3) [D, Theorem 3.5]. Suppose $m \geq 3$. The abstract $m$-type ( $G$, $\left.\pi_{m}(X), r\right)$ is the algebraic $m$-type of some $(G, m)$-complex if, and only if, $\nu(r)=$ $[r, N]=0$, that is $[r, N]$ is stably free.

Consequently, the $k$-invariants $r$ which can arise from $(G, m)$-complexes with algebraic $m$-type $\left(G, \pi_{m}(X), r\right)$ form a subgroup of $Z_{n}^{*}$. In particular $\left(G, \pi_{m}(X), 1\right)$ is the algebraic $m$-type of a $(G, m)$-complex (namely $\left.X\right)$.

Let $G$ be a finite group of order $n$ such that there is a $(G, m)$-complex $X$. As in [D] we make the definitions

$$
Q_{m}\left(\pi_{m}(X)\right)=\left\{r \in Z_{n}^{*} \subset H^{m+1}\left(G, \pi_{m}(X)\right) \mid\left(G, \pi_{m}(X), 1\right) \cong\left(G, \pi_{m}(X), r\right)\right\},
$$

where $\cong$ is isomorphism as abstract $m$-types, and

$$
F(G)=\left\{r \in Z_{n}^{*} \mid(r, N) \text { is free }\right\} .
$$

Suppose now that $G$ has periodic cohomology and minimal free period $k$. The following is a consequence of [D, Corollary (8.4), (a), p. 275].

Theorem (2.4). $F(G) \subset Q_{k}(A[G])$.

\section{PROOF OF THE MAIN THEOREM}

Theorem (3.1). Suppose $p: \widetilde{M} \rightarrow M$ is a nontrivial regular covering with $M$ and $\widetilde{M}$ homology 3-spheres. Then the group of covering transformations is the binary icosahedral group $I^{*}$.

Proof. Let $G$ be the group of covering transformations of $p: \widetilde{M} \rightarrow M$. Then $G$ has periodic cohomology and its period is either 2 or 4 . From the exact sequence $1 \rightarrow \pi_{1}(\widetilde{M}) \stackrel{p_{*}}{\rightarrow} \pi_{1}(M) \rightarrow \pi_{1}(M) / p_{*}\left(\pi_{1}(\widetilde{M})\right) \cong G \rightarrow 1$ we see that $G$ is perfect. Therefore $G \cong I^{*}$ (see [Sj]). Q.E.D.

Corollary (3.2). Let $M$ be a homology 3-sphere such that $\pi_{1}(M)$ has a subgroup of finite index which is not a divisor of 120 . Then there is a sequence $\left\{G_{i}\right\}_{i=1,2 \ldots}$ of subgroups of finite index in $\pi_{1}(M)$ with $G_{i+1} \subsetneq G_{i}, i=1,2, \ldots$.

Proof. Let $\tilde{G}_{1} \subset \pi_{1}(M)$ be a subgroup of finite index which is not a divisor of 120. Let $\pi_{1}(M)=g_{0} \widetilde{G}_{1} \cup \cdots \cup g_{k} \widetilde{G}_{1}$ be the left coset decomposition of 


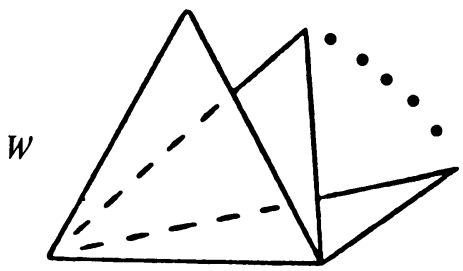

$\widetilde{M}$
120 copies of $C \tilde{M}$ joined along $\tilde{M}$

\section{Figure 1}

$\pi_{1}(M)$. Then $G_{1}=\bigcap_{i=0}^{k} g_{i} \widetilde{G}_{1} g_{i}^{-1}$ is a normal subgroup of $\pi_{1}(M)$ with index $\left(G_{1}: \pi_{1}(M)\right)=\operatorname{index}\left(G_{1}: \widetilde{G}_{1}\right) \cdot \operatorname{index}\left(\widetilde{G}_{1}: \pi_{1}(M)\right)$. Hence index $\left(G_{1}: \pi_{1}(M)\right)$ does not divide 120. Let $p: M_{1} \rightarrow M$ be the covering with $p_{*} \pi_{1}\left(M_{1}\right)=G_{1}$. By Theorem 3.1, $M_{1}$ cannot be a homology 3-sphere. Let

$$
\widetilde{G}_{2}=p_{*} \operatorname{ker}\left(\pi_{1}\left(M_{1}\right) \rightarrow H_{1}\left(M_{1}\right) \rightarrow \text { onto finite abelian group } \neq 0\right) .
$$

Then index $\left(\widetilde{G}_{2}: \pi_{1}\left(M_{1}\right)\right)$ does not divide 120 and the construction can be continued. Q.E.D.

If $X$ is a space and $f: X \rightarrow Y$ is a map let $C X, S X$ and $C_{f}$ denote the unreduced cone, suspension and mapping cone, respectively.

Now let $M, \widetilde{M}$ be homology 3-spheres and let $p: \widetilde{M} \rightarrow M$ be a regular covering with $I^{*}$ as group of covering transformations. Define $W=I^{*} \times$ $C \widetilde{M} /(g, \tilde{x}, 0) \sim(h, \tilde{x}, 0)$. See Figure 1 .

Note that $W$ is 3-connected since collapsing one of the cones to a point gives a homotopy equivalence

$$
W \simeq \underbrace{S \widetilde{M} \vee \cdots \vee \widetilde{M}}_{119 \text { copies }} \simeq \underbrace{S^{4} \vee \cdots \vee S^{4}}_{119 \text { copies }}
$$

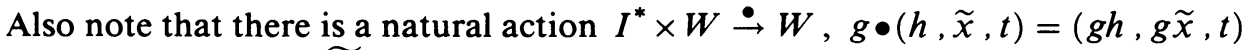
and that $W / I^{*}=C \widetilde{M} /(\widetilde{x}, 0) \sim(g \tilde{x}, 0)=C_{p}$. Since this action is fixed point free this implies that $W$ is the universal covering space of $C_{p}$.

Lemma (3.3). $C_{p}$ is an $\left(I^{*}, 4\right)$-complex whose algebraic 4-type is $\left(I^{*}, A\left[I^{*}\right], r\right)$ for some $r \in Z_{120}^{*}$.

Proof. The only part requiring proof is that $\pi_{4}\left(C_{p}\right) \cong A\left[I^{*}\right]$ as (left) $Z\left[I^{*}\right]$ modules. Thus consider the following portion of the homology exact sequence of the pair $(W, \widetilde{M})$ :

$$
0 \rightarrow H_{4}(W) \rightarrow H_{4}(W, \widetilde{M}) \stackrel{\partial}{\rightarrow} H_{3}(\widetilde{M}) \rightarrow 0 .
$$

This is an exact sequence of $Z\left[I^{*}\right]$-modules with $H_{4}(W) \cong \pi_{4}\left(C_{p}\right)$ as $Z\left[I^{*}\right]$ modules and $H_{3}(\widetilde{M}) \cong Z$ as a trivial $Z\left[I^{*}\right]$-module.

Let $U=\left\{(g, \tilde{x}, t) \in W \mid t \leq \frac{1}{2}\right\}$. See Figure 2 . 


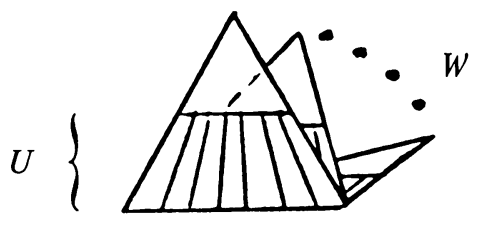

$\widetilde{M}$

FIGURE 2

Then we have the following isomorphism of $Z\left[I^{*}\right]$-modules:

$$
\begin{aligned}
H_{4}(W, \widetilde{M}) & \cong H_{4}(W, U) \cong H_{4}(W-\operatorname{int} U, U-\operatorname{int} U) \cong H_{4}\left(I^{*} \times(C \widetilde{M}, \widetilde{M})\right) \\
& \cong Z\left[I^{*}\right] \quad \text { since } H_{4}(C \widetilde{M}, \widetilde{M}) \cong Z .
\end{aligned}
$$

With respect to these isomorphisms the boundary homomorphism

$$
\partial: H_{4}(W, \widetilde{M}) \rightarrow H_{3}(\widetilde{M})
$$

is just the augmentation homomorphism and therefore $\pi_{4}\left(C_{p}\right) \cong A\left[I^{*}\right]$ as $Z\left[I^{*}\right]$-modules. Q.E.D.

Theorem (3.4). Up to homotopy there is only one $\left(I^{*}, 4\right)$-complex $X$ such that $\pi_{4}(X) \cong A\left[I^{*}\right]$. In particular, if $p: \widetilde{M} \rightarrow M$ is a nontrivial regular covering of a homology 3-sphere $\widetilde{M}$ onto the homology 3-sphere $M$, then $C_{p}$ is homotopy equivalent to $C_{q}$, where $q: S^{3} \rightarrow D^{3}$ is the universal covering.

Proof. According to $(3.3), C_{p}$ and $C_{q}$ are $\left(I^{*}, 4\right)$ complexes with $\pi_{4} \cong A\left[I^{*}\right]$. If $X$ is any $\left(I^{*}, 4\right)$ complex with algebraic 4-type $\left(I^{*}, A\left[I^{*}\right], r\right)$, then $[r, N]=$

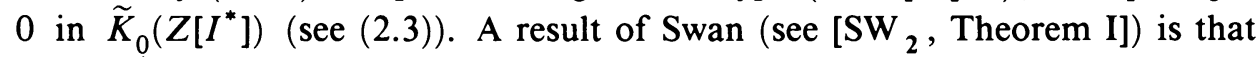
$r \in F\left(I^{*}\right)$. Then from (2.4) we see that there is only one isomorphism class of algebraic 4-types $\left(I^{*}, A\left[I^{*}\right], r\right)$. Using (2.2) we now have that $C_{p}$ is homotopy equivalent to $C_{q}$. Q.E.D.

Theorem (3.5). Let $M, \widetilde{M}$ be homology 3-spheres and $p: \widetilde{M} \rightarrow M$ a nontrivial regular covering. Then there is a map $f: M \rightarrow D^{3}$ onto the dodecahedral space $D^{3}$ such that $f_{*}\left(\pi_{1}(M)\right)=\pi_{1}\left(D^{3}\right)$, the degree of $f$ is relatively prime to 120 , and $p_{*}\left(\pi_{1}(\widetilde{M})\right)=\operatorname{ker}\left(f_{*}: \pi_{1}(M) \rightarrow \pi_{1}\left(D^{3}\right)\right)$.

Proof. By (3.4) there is a homotopy equivalence $h: C_{p} \rightarrow C_{q}$. Let $i: M \rightarrow C_{p}$ and $j: D^{3} \rightarrow C_{q}$ be the inclusions. Then we can alter $h$ by a homotopy, if necessary, so that $h i(M) \subset D^{3}$. Let $f=h i: M \rightarrow D^{3}$. Thus we have the commutative diagram

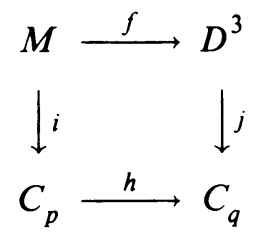

The map $f$ has the desired properties. Q.E.D. 
Theorems (3.1), (3.4) and (3.5) prove the Main Theorem. It should be pointed out that one can construct such a map $f: M \rightarrow D^{3}$ by elementary obstruction theory. In fact the regular covering $p: \widetilde{M} \rightarrow M$ induces an epimorphism $\theta: \pi_{1}(M) \rightarrow I^{*}$ and $f$ can be chosen so that $\theta$ corresponds to $f_{*}: \pi_{1}(M) \rightarrow \pi_{1}\left(D^{3}\right)$. The map $f: M \rightarrow D^{3}$ lifts to a map $\tilde{f}: \widetilde{M} \rightarrow S^{3}$, and this will then produce a map $h: C_{p} \rightarrow C_{q}$ by coning. However, $h$ will not in general be a homotopy equivalence.

Theorem (3.5) raises the following open question.

Question. Suppose $M, \widetilde{M}$ are homology 3-spheres and $p: \widetilde{M} \rightarrow M$ is a nontrivial regular covering. Then is there a degree 1 map $f: M \rightarrow D^{3}$ such that $\left.p_{*}\left(\pi_{1}(\widetilde{M})\right)=\operatorname{ker}\left(f_{*}: \pi_{1}(M)\right) \rightarrow \pi_{1}\left(D^{3}\right)\right) ?$

It follows from [Ol] that for each integer $m$ there is a map $h_{m}: D^{3} \rightarrow D^{3}$ that induces the identity on $\pi_{1}\left(D^{3}\right)$ and that has degree $1+120 \mathrm{~m}$. The binary icosahedral group $I^{*}$ has only one nontrivial outer automorphism $\alpha: I^{*} \rightarrow I^{*}$. There is a map $h_{\alpha}: D^{3} \rightarrow D^{3}$ that induces $\alpha$ on $\pi_{1}\left(D^{3}\right)$ and that has degree 49 [Pl]. Composing the map $f: M \rightarrow D^{3}$ with maps of the types $h_{m}, h_{\alpha}$, we see that we can alter the degree of the map $f$ to $\operatorname{deg} f+120 m$, or to 49 $\operatorname{deg} f+120 m$.

Suppose that $p: \widetilde{M} \rightarrow M$ is a (regular) covering of the homology 3-sphere $\widetilde{M}$ onto the homology 3-sphere $M$ (e.g. $q: S^{3} \rightarrow D^{3}$ ). Let $X$ be an arbitrary homology 3-sphere and let $n$ be the number of points in a fiber $p^{-1}(x), x \in M$ ( $n=120$ if the covering is regular and nontrivial). Then $p: \widetilde{M} \rightarrow M$ extends to a (regular) covering $\hat{p}: \widetilde{M} \# n X \rightarrow M \# X$ of connected sums, where $M \# X$ is the connected sum defined by removing a 3-cell $E^{3} \subset M$ from $M$, a 3-cell from $X$, and identifying their boundaries, and $\widetilde{M} \# n X$ is defined by removing the $n$ 3-cells $p^{-1}\left(E^{3}\right)$ from $\widetilde{M}$ and sewing in $n$ copies of $\overline{X \text {-(3-cell) }}$. See Figure 3. The connected sums $M \# X$ and $\widetilde{M} \# n X$ are homology 3-spheres.

Theorem (3.6). Let $\widetilde{M}, M$ be homology 3-spheres and $p: \widetilde{M} \rightarrow M$ a nontrivial regular covering. Suppose that $M$ is not irreducible. Then there are irreducible homology 3-spheres $\widetilde{M}_{0}, M_{0} ;$ a nontrivial regular covering $p_{0}: \widetilde{M}_{0} \rightarrow M_{0}$; and a homology 3-sphere $X$ so that $M=M_{0} \# X, \widetilde{M}=\widetilde{M}_{0} \# 120 X$, and $p=\hat{p}_{0}$.

Proof. Since $M$ is not irreducible we have $M=M_{0} \# M_{1} \# \cdots \# M_{k}$ with $M_{0}$, $\ldots, M_{k}$ irreducible homology 3-spheres. The covering $p: \widetilde{M} \rightarrow M$ defines canonical coverings $p_{i}: \widetilde{M}_{i} \rightarrow M_{i}, i=0, \ldots, k$. The components of $\widetilde{M}_{i}$ must be homology 3-spheres. If $M_{i}^{\prime} \subset \widetilde{M}_{i}$ is a component, then $p_{i} \mid: M_{i}^{\prime} \rightarrow M_{i}$ is a covering. There must exist at least one $i$ and one component $M_{i}^{\prime} \subset \widetilde{M}_{i}$ such that $p_{i} \mid: M_{i}^{\prime} \rightarrow M_{i}$ is nontrivial (otherwise, replacing each $M_{i}^{\prime}$ by a 3 -sphere we can construct a nontrivial covering $S^{3} \rightarrow S^{3}=S_{0}^{3} \# \ldots \# S_{k}^{3}$, a contradiction). Suppose $p_{0} \mid: M_{0}^{\prime} \rightarrow M_{0}$ is nontrivial. Define $X$ to be $M_{1} \# \cdots \# M_{k}$. Then 


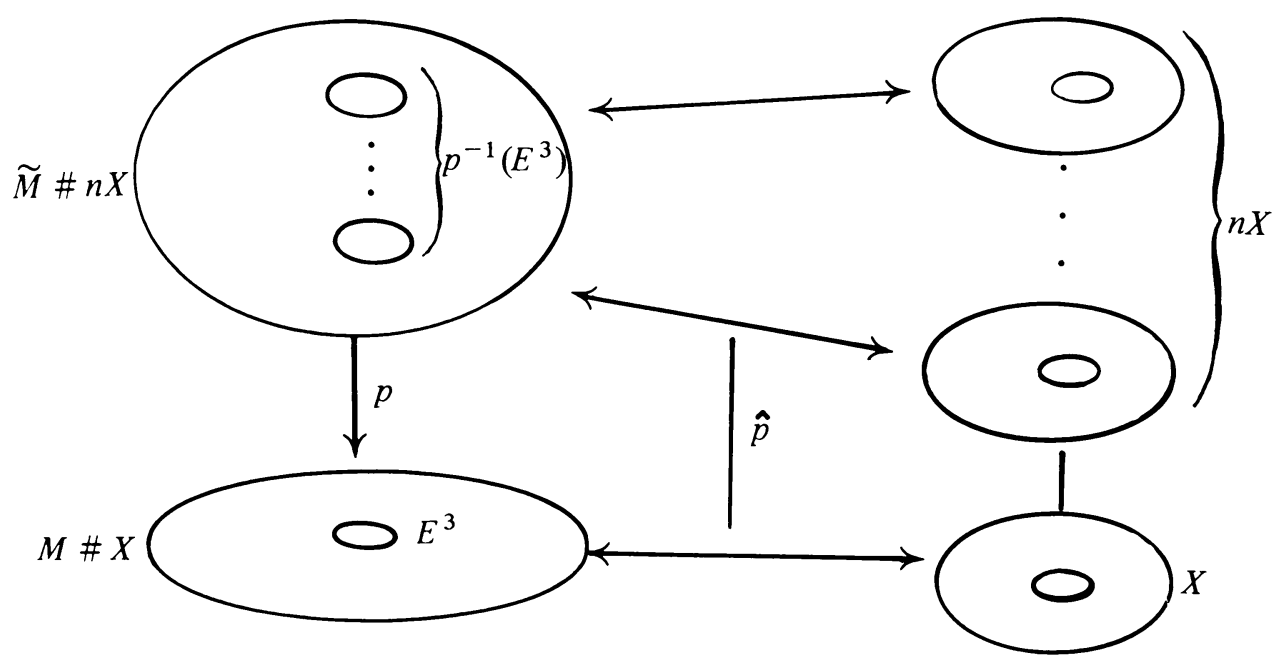

FigURE 3

$M=M_{0} \# X$. Note that $p_{0} \mid: M_{0}^{\prime} \rightarrow M_{0}$ is a regular covering. By Theorem 3.1, both $p: \widetilde{M} \rightarrow M$ and $p_{0} \mid: M_{0}^{\prime} \rightarrow M_{0}$ are 120-sheeted. Therefore $M_{0}^{\prime}=\widetilde{M}_{0}$. Since $\widetilde{M}$ is a homology 3 -sphere, $p^{-1}(X)$ consists of 120 copies of $X$. Q.E.D.

\section{Regular coverings of Seifert Fibered homology 3-SPHeres BY HOMOLOGY 3-SPHERES}

We have the following uniqueness result.

Theorem (4.1). Let $M$ be a homology 3-sphere that admits a Seifert fibration and a nontrivial regular covering $p: \widetilde{M} \rightarrow M$ by a homology 3-sphere. Then necessarily $M=D^{3}$ and $\widetilde{M}=S^{3}$.

Proof. Let $\left(\alpha_{1}, \beta_{1}\right), \ldots,\left(\alpha_{r}, \beta_{r}\right)$ be the Seifert invariants of the Seifert fibration of $M$. By Satz 12 of [S] we must have $r \geq 3$ and $\alpha_{1}, \ldots, \alpha_{r}$ relatively prime in pairs. We give $\widetilde{M}$ the Seifert fibration induced by the covering $p: \widetilde{M} \rightarrow M$.

If $S_{0}^{1} \subset M$ is a regular fiber, then the components of $p^{-1}\left(S_{0}^{1}\right)$ are all regular fibers. If $S^{1} \subset M$ is a singular fiber, we claim that the components of $p^{-1}\left(S^{1}\right)$ must also be regular fibers. To prove this suppose $\widetilde{S}^{1} \subset p^{-1}\left(S^{1}\right)$ is a singular fiber. First we show that $\widetilde{S}^{1}=p^{-1}\left(S^{1}\right)$. Otherwise there is another component $\tilde{S}_{1}^{1} \subset p^{-1}\left(S^{1}\right)$. Then, since the group of covering transformations of the regular covering $p: \widetilde{M} \rightarrow M$ acts transitively on $p^{-1}\left(S^{1}\right), \widetilde{S}^{1}$ and $\widetilde{S}_{1}^{1}$ must have the same Seifert invariants. Since $\widetilde{M}$ is a homology 3 -sphere this contradicts Satz 12 of $[\mathrm{S}]$. Therefore $\tilde{S}^{1}=p^{-1}\left(S^{1}\right)$. This now contradicts the fact that the group of covering transformations is the noncyclic group $I^{*}$.

Thus the Seifert fibration of $\widetilde{M}$ has no singular fibers. By the remark preceding Satz 12 of [S], $\widetilde{M}$ must be the 3-sphere. Therefore the fundamental group 
of $M$ must be finite. Again by Satz 12 of [S], $M$ must be the dodecahedral space $D^{3}$. Q.E.D.

\section{EXAMPLES OF REGULAR COVERINGS OF IRREDUCIBLE HOMOLOGY 3-SPHERES BY HOMOLOGY 3-SPHERES}

We present two methods of constructing regular coverings $p: \widetilde{M} \rightarrow M$ such that $M, \widetilde{M}$ are irreducible homology 3-spheres.

Theorem (5.1). Let $p_{0}: \widetilde{M}_{0} \rightarrow M_{0}$ be a regular covering of the irreducible homology 3-sphere $M_{0}$ by the homology 3-sphere $\widetilde{M}_{0}$. Then there is a sufficiently large homology 3-sphere $M$ containing an incompressible torus, $M$ and $M_{0}$ not homotopy equivalent, a regular covering $p: \widetilde{M} \rightarrow M$ of $M$ by a homology 3-sphere, and there are degree 1 maps $h: M \rightarrow M_{0}, \widetilde{h}: \widetilde{M} \rightarrow \widetilde{M}_{0}$ such that the following diagram

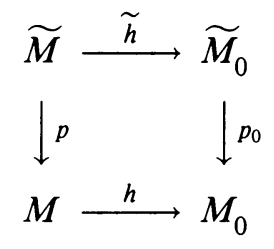

commutes.

Proof. Let $W$ be an irreducible orientable compact 3-manifold with $\partial W$ a torus, $H_{1}(W)=\mathbf{Z}$, and $W$ not a solid torus (e.g. let $X$ be any irreducible homology 3-sphere with $\pi_{1}(X) \neq 1$, and $S^{1} \subset X$ a 1-sphere which is not nullhomotopic in $X$; or $X=S^{3}$ and $S^{1} \subset S^{3}$ a nontrivial knot. Then $W=\overline{X-N\left(S^{1}\right)}$, where $N\left(S^{1}\right)$ is a regular neighbourhood of $S^{1}$ in $X$, is an irreducible orientable compact 3-manifold with $\partial W$ a torus, $H_{1}(W)=\mathbf{Z}$, and $W$ is not a solid torus). Note that $\partial W$ is incompressible in $W$. By a standard argument there is a proper surface $F \subset W$ with $F \cap \partial W=\partial F$ a 1-sphere. Let $\partial W=S^{1} \times \partial F$ be a representation such that $\left[S^{1}\right]$ is a generator of $H_{1}(W)=\mathbf{Z}$.

By a result of [Ha] there is a 1-sphere $S_{0}^{1} \subset M_{0}$ which is nullhomotopic in $M_{0}$ and such that $C=\overline{M_{0}-N\left(S_{0}^{1}\right)}$ is a fiber bundle over a 1-sphere with fiber a surface $F_{0}$, where $N\left(S_{0}^{1}\right)=S_{0}^{1} \times D_{0}^{2}$ is a regular neighbourhood of $S_{0}^{1}$ in $M_{0}$. Applying the exact Mayer-Vietoris sequence of the pair $\left(C, N\left(S_{0}^{1}\right)\right)$, we may assume that $\left[\partial F_{0}\right)=\left[S^{1}\right]$ in $H_{1}\left(\partial N\left(S_{0}^{1}\right)\right)$. Note that $C$ is irreducible and that the torus $\partial C$ is incompressible in $C$. Let $g:(W, \partial W) \rightarrow\left(N\left(S_{0}^{1}\right), \partial N\left(S_{0}^{1}\right)\right)$ be a map such that $g \mid: \partial W \rightarrow \partial N\left(S_{0}^{1}\right)$ is an isomorphism with $g(F)=D_{0}^{2}$ and $g_{*}\left[S^{1}\right]=\left[S_{0}^{1}\right]$ in $H_{1}\left(\partial N\left(S_{0}^{1}\right)\right)$. Define

$$
M=C \cup W / x=g(x), x \in \partial C,
$$




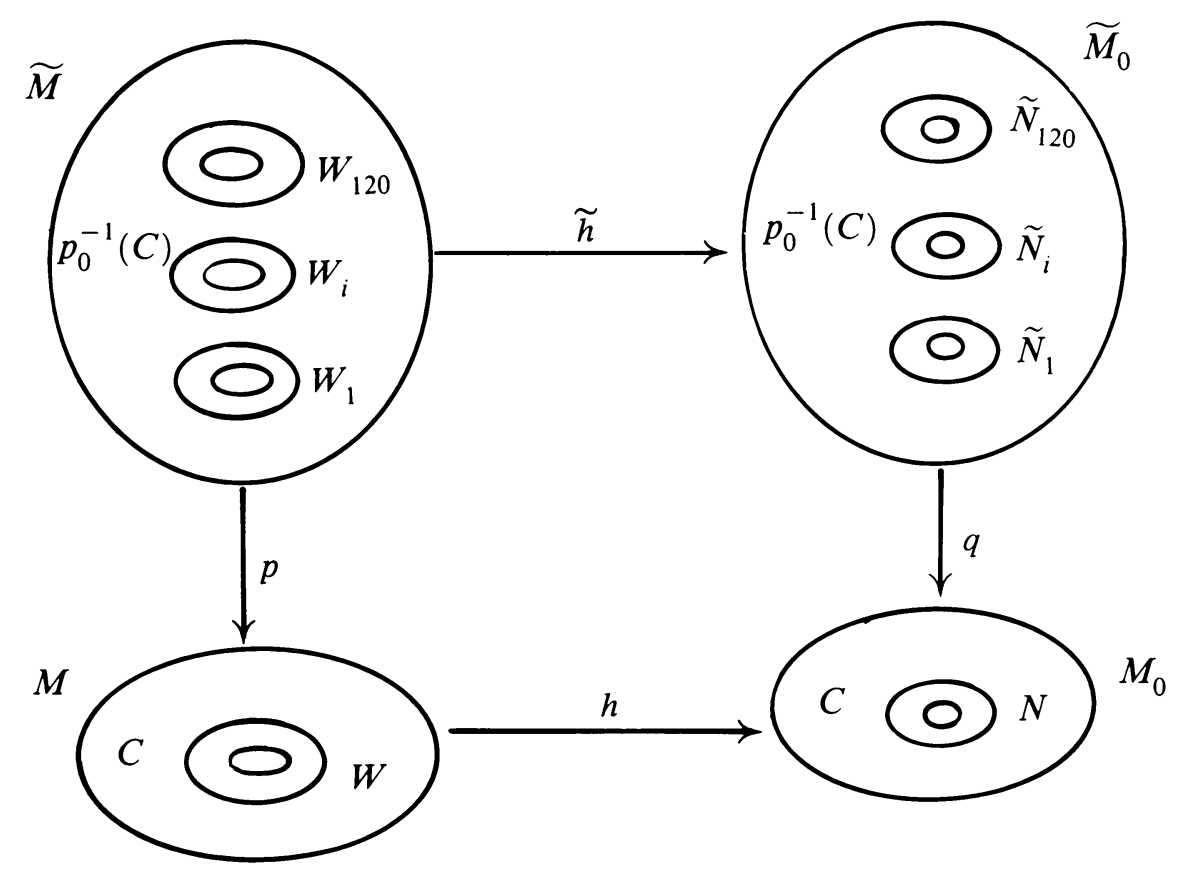

FIGURE 4

and the map $h: M \rightarrow M_{0}$ by

$$
h(x)= \begin{cases}x, & x \in C, \\ g(x), & x \in W\end{cases}
$$

The closed 3-manifold $M$ is orientable, irreducible and the torus $\partial W=\partial C$ is incompressible in $M$. From the exact Mayer-Vietoris sequence of the pair $(C, W)$ it follows that $M$ is a homology 3-sphere. The map $h: M \rightarrow M_{0}$ has degree 1. By construction $N=N\left(S_{0}^{1}\right) \subset M_{0}$ is nullhomotopic and therefore $p_{0}^{-1}(N)$ consists of 120 copies $\widetilde{N}_{1}, \ldots, \widetilde{N}_{120}$ of $N$. Now take 120 copies $W_{i}$ of $W, i=1, \ldots, 120$. Define: $\widetilde{M}=\bigcup_{i=1}^{120} W_{i} \cup p_{0}^{-1}(C)$. See Figure 4. The map $h: M \rightarrow M_{0}$ lifts to a degree 1 map $\widetilde{h}: \widetilde{M} \rightarrow \widetilde{M}_{0}$. A Mayer-Vietoris sequence applied to the map $h:\left(\widetilde{M}, p^{-1}(W), p^{-1}(C)\right) \rightarrow\left(M_{0}, p_{0}^{-1}(N), p_{0}^{-1}(C)\right)$ proves that $\widetilde{M}$ is a homology 3 -sphere. Finally, $M$ and $M_{0}$ cannot be homotopy equivalent since $\pi_{1}(M)$ and $\pi_{1}\left(M_{0}\right)$ cannot be isomorphic. Namely, $h_{*}: \pi_{1}(M) \rightarrow \pi_{1}\left(M_{0}\right)$ is an epimorphism with $\operatorname{ker}\left(h_{*}\right) \neq 1$. If $\pi_{1}(M) \cong$ $\pi_{1}\left(M_{0}\right)$, then $\pi_{1}(M) \cong \pi_{1}(M) / \operatorname{ker}\left(h_{*}\right)$ and $\pi_{1}(M)$ is not Hopfian. But $M$ is sufficiently large and therefore $\pi_{1}(M)$ is residually finite and hence Hopfian, a contradiction. Q.E.D.

Starting with the regular covering $q: S^{3} \rightarrow D^{3}$ we can thus construct an abundance of sufficiently large homology 3 -spheres containing incompressible tori that admit regular coverings by homology 3 -spheres. 
For the second construction we utilize the Seifert fibration of $D^{3}$. Let $q: S^{3} \rightarrow D^{3}$ be the universal covering of the dodecahedral space $D^{3}$. Then $q$ lifts the Seifert fibration of $D^{3}$ to a Seifert fibration of $S^{3}$. The group of covering transformations acts equivariantly on the fibers of the induced Seifert fibration.

Recall that the binary icosahedral group $\pi_{1}\left(D^{3}\right)$ has order 120 and that its center is a cyclic group of order 2. Since each regular fiber $S_{0}^{1} \subset D^{3}$ defines a generator $\left[S_{0}^{1}\right] \in \pi_{1}\left(D^{3}\right)$ of the center, $q^{-1}\left(S_{0}^{1}\right)$ has 60 components. If $\tilde{S}_{0}^{1} \subset q^{-1}\left(S_{0}^{1}\right)$ is a component, it is a regular fiber with $q \mid: \tilde{S}_{0}^{1} \rightarrow S_{0}^{1}$ a 2-sheeted covering.

We complete the description of $q: S^{3} \rightarrow D^{3}$ as follows. Let $S_{\alpha}^{1} \subset D^{3}$ be a singular fiber with Seifert invariant $(\alpha, 1), \alpha=2,3,5$. Let $\widetilde{S}^{1} \subset q^{-1}\left(S_{\alpha}\right)$ be a component and suppose that it has Seifert invariant $(\widetilde{\alpha}, \widetilde{\beta})$. Assume that $q \mid: \widetilde{S}^{1} \rightarrow S_{\alpha}^{1}$ is a $\sigma$-sheeted covering. Then by the remarks on p. 196 of [S] we have $\widetilde{\alpha}=\alpha /(\alpha, \sigma)$. Now $(\alpha, \sigma)=1$ is not possible, since otherwise $\widetilde{\alpha}=$ $\alpha$. But then, since $\pi_{1}\left(D^{3}\right)$ acts transitively on $q^{-1}\left(S_{\alpha}^{1}\right)$, the induced Seifert fibration of $S^{3}$ has more than one fiber with the same Seifert invariants. A contradiction to Satz 12 of [S]. Therefore $(\alpha, \sigma)=\alpha$ and hence $\widetilde{\alpha}=1$. Thus each fiber $\widetilde{S}^{1} \subset q^{-1}\left(S_{\alpha}^{1}\right)$ is regular. The Seifert fibration induced on $S^{3}$ has no singular fibers; therefore it is the Hopf fibration [S].

Now let $F$ be a closed orientable surface, $B \subset F$ a 2-cell, and $\phi: F \rightarrow F$ an orientation preserving isomorphism such that $\phi(x)=x$ for all $x \in B$. Define

$$
\begin{aligned}
& M_{\phi}=F \times[0,1] /(x, 0) \sim(\phi(x), 1), \\
& \pi: M_{\phi} \rightarrow S^{1}=[0,1] / 0 \sim 1 \quad \text { by } \pi(x, t)=t .
\end{aligned}
$$

Then $M_{\phi}$ is a bundle over $S^{1}$ with fiber $F$ and bundle map $\pi$. An application of a Mayer-Vietoris sequence gives the following exact sequence in homology.

$$
0 \rightarrow H_{1}(F) /\left(\phi_{*}-\mathrm{id}\right) H_{1}(F) \stackrel{i_{*}}{\rightarrow} H_{1}\left(M_{\phi}\right) \stackrel{\pi_{*}}{\rightarrow} H_{1}\left(S^{1}\right)=Z \rightarrow 0 .
$$

Here $\bar{l}_{*}$ is the map induced by the inclusion $l: F \rightarrow M_{\phi}, l(x)=(x, 0)$. Thus $H_{1}\left(M_{\phi}\right) \cong Z \oplus \operatorname{coker}\left(\phi_{*}-\mathrm{id}\right)$. In a similar fashion we define

$$
W_{\phi}=(\overline{F-B}) \times[0,1] /(x, 0) \sim(\phi(x), 1)=\overline{M_{\phi}-B \times S^{1}} .
$$

Again we have $H_{1}\left(W_{\phi}\right) \cong Z \oplus \operatorname{coker}\left(\phi_{*}-\right.$ id $)$. In particular, if $\phi_{*}-$ id is invertible it follows that $H_{1}\left(W_{\phi}\right) \cong Z$ with generator $\left[S^{1}\right]$.

In the dodecahedral space $D^{3}=S_{4}^{2} \times S^{1} \cup_{h_{0}} B_{0}^{\prime} \times S^{1} \cup \cdots \cup_{h_{3}} B_{3}^{\prime} \times S^{1}$ let $B \subset \operatorname{int} S_{4}^{2}$ be a 2 -cell and let $D_{0}^{3}=\overline{D^{3}-B \times S^{1}}$. Then $H_{1}\left(D_{0}^{3}\right) \cong Z$ with generator $[\partial B]$.

Define $M(\phi)=W_{\phi} \cup_{\partial} D_{0}^{3}$ by identifying the boundary tori $\partial B \times S^{1}$ of $W$ and $D_{0}^{3}$ as suggested by the notation. Notice that $M(\phi)$ is irreducible (by (2.1)) and contains the incompressible torus $\partial B \times S^{1}$. We have $H_{1}(M(\phi)) \cong \operatorname{coker}\left(\phi_{*}-\mathrm{id}\right)$ 
since $[\partial B]=0$ in $H_{1}\left(W_{\phi}\right)$ and $\left[S^{1}\right]=0$ in $H_{1}\left(D_{0}^{3}\right)$. Therefore, if $\phi_{*}-$ id is invertible it follows that $M(\phi)$ is a homology 3-sphere.

Next we construct a degree 1 map $h: M(\phi) \rightarrow D^{3}$. Let $h \mid D_{0}^{3}=$ id. The isomorphism id: $\partial W_{\phi}=\partial B \times S^{1} \rightarrow \partial D_{0}^{3}=\partial B \times S^{1}$ extends to a map $W_{\phi} \rightarrow$ $B \times S^{1}$ by mapping a collar $\overline{F-B} \times[-\varepsilon, \varepsilon]$ onto a collar $B \times[-\varepsilon, \varepsilon]$ and then extending this map to a map of $\overline{\left(W_{\phi}-\overline{F-B}\right) \times[-\varepsilon, \varepsilon]}$ onto the 3-cell $\overline{B \times S^{1}-B \times[-\varepsilon, \varepsilon] \text {. }}$

Summarizing, we have for each orientation preserving isomorphism $\phi:(F, B)$ $\rightarrow(F, B)$, where $\phi=$ id on the 2-cell $B$, constructed a 3-manifold $M(\phi)$ and a degree 1 map $h: M(\phi) \rightarrow D^{3}$. Moreover, $M(\phi)$ is irreducible, contains an incompressible torus, and is a homology 3-sphere if, and only if, $\phi_{*}-\mathrm{id}: H_{1}(F) \rightarrow H_{1}(F)$ is invertible.

Our goal is to find conditions on $\phi$ which will ensure that the covering of $M(\phi)$ induced by $h$ from the universal covering $q: S^{3} \rightarrow D^{3}$ will also be a homology 3-sphere. Let $\widetilde{M(\phi)}$ denote this covering.

Let $d: S^{1} \rightarrow S^{1}$ be the 2-sheeted covering of $S^{1}$ and let $d: M_{\phi^{2}} \rightarrow M_{\phi}$ be the corresponding 2-sheeted fiber preserving covering. If we use the notation $[x, t]$ for a typical point then the coordinate description of $d$ is

$$
d[x, t]= \begin{cases}{[\phi(x), 2 t]} & \text { if } 0 \leq t \leq 1 / 2 \\ {[x, 2 t-1]} & \text { if } 1 / 2 \leq t \leq 1\end{cases}
$$

Then $d^{-1}\left(B \times S^{1}\right)=B \times \widetilde{S}^{1}$ and $d: B \times \widetilde{S}^{1} \rightarrow B \times S^{1}$ is a 2-sheeted covering with $d_{*}[\partial B]=[\partial B]$ and $d_{*}\left[\tilde{S}^{1}\right]=2\left[S^{1}\right]$ in $H_{1}\left(\partial B \times S^{1}\right)$. Therefore we can induce a 2-sheeted covering $d: W_{\phi^{2}} \rightarrow W_{\phi}$.

From the description of $q: S^{3} \rightarrow D^{3}$ we see that $q^{-1}\left(B \times S^{1}\right)$ consists of 60 distinct solid tori $B \times \widetilde{S}_{i}^{1}, 1 \leq i \leq 60$, and that $q: B \times \widetilde{S}_{i}^{1} \rightarrow B \times S^{1}$ is a 2-sheeted covering satisfying $q_{*}[\partial B]=[\partial B], q_{*}\left[\widetilde{S}_{i}^{1}\right]=2\left[S^{1}\right]$ in $H_{1}\left(\partial B \times S^{1}\right)$. Now take 60 copies $\widetilde{W}_{i}, 1 \leq i \leq 60$, of $W_{\phi^{2}}$ and define $\widetilde{M}=\left(\bigcup_{i=1}^{60} \widetilde{W}_{i}\right) \cup_{\partial} q^{-1}\left(D_{0}^{3}\right)$, where we identify the boundary torus $\partial \widetilde{W}_{i}=\partial B \times \widetilde{S}_{i}^{1}$ of $\widetilde{W}_{i}$ with the boundary torus $\partial B \times \widetilde{S}_{i}^{1}$ of $q^{-1}\left(D_{0}^{3}\right)$ as suggested by the notation, $1 \leq i \leq 60$.

Now define a covering projection $p: \widetilde{M} \rightarrow M(\phi)$ by the formulas: $p \mid q^{-1}\left(D_{0}^{3}\right)$ $=q\left|q^{-1}\left(D_{0}^{3}\right), \quad p\right| \widetilde{W}_{i}=d \mid \widetilde{W}_{i}, 1 \leq i \leq 60$. This definition is valid since on $q^{-1}\left(D_{0}^{3}\right) \cap \widetilde{W}_{i}=\partial B \times \widetilde{S}_{i}$ the maps $q$ and $d$ agree.

The map $h:\left(W_{\phi}, \partial W_{\phi}\right) \rightarrow\left(B \times S^{1}, \partial B \times S^{1}\right)$ lifts to a map $\widetilde{h}_{i}:\left(\widetilde{W}_{i}, \partial \widetilde{W}_{i}\right) \rightarrow$ $\left(B \times \widetilde{S}_{i}^{1}, \partial B \times \widetilde{S}_{i}^{1}\right)$ which is the identity on the boundary torus $\partial \widetilde{W}_{i}, 1 \leq i \leq 60$, and which makes the following diagram commute:

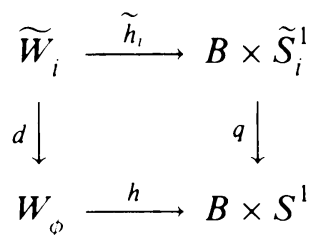


Thus we can define $\widetilde{h}: \widetilde{M} \rightarrow S^{3}$ by

$$
\widetilde{h}\left|q^{-1}\left(D_{0}^{3}\right)=\mathrm{id}, \quad \widetilde{h}\right| \widetilde{W}_{i}=\widetilde{h}_{i}, \quad 1 \leq i \leq 60 .
$$

Then $\widetilde{h}$ has degree 1 and is a lift of $h: M(\phi) \rightarrow D^{3}$.

It follows that $p: \widetilde{M} \rightarrow M(\phi)$ is the covering $\widetilde{M}(\phi) \rightarrow M(\phi)$ induced from $q: S^{3} \rightarrow D^{3}$ by $h: M(\phi) \rightarrow D^{3}$.

Finally we compute $H_{1}(M(\phi))$. To do this we apply a Mayer-Vietoris sequence to $M(\phi)=\left(\bigcup_{i=1}^{60} \widetilde{W}_{i}\right) \cup_{\partial} q^{-1}\left(D_{0}^{3}\right)$. Note that $H_{1}\left(q^{-1}\left(D_{0}^{3}\right)\right) \cong 60 Z$ with generators $\left[\partial B_{i}\right], 1 \leq i \leq 60$, and $H_{1}\left(\bigcup_{i=1}^{60} \widetilde{W}_{i}\right) \cong 60 H_{1}\left(W_{\phi^{2}}\right) \cong 60 Z \oplus$ $60 \operatorname{coker}\left(\phi_{*}^{2}-\mathrm{id}\right)$, with generators $\left[\tilde{S}_{i}^{1}\right], 1 \leq i \leq 60$, for the free summand. It follows that $H_{1}(\widetilde{M(\phi)}) \cong 60 \operatorname{coker}\left(\phi_{*}^{2}-\right.$ id $)$.

The following theorem summarizes the results of the above construction.

Theorem (5.2). Suppose $F$ is a closed orientable surface and $\phi: F \rightarrow F$ is an orientation preserving isomorphism which is the identity on some 2-cell $B \subset F$. Let $M(\phi)=\{(\overline{F-B}) \times[0,1] /(x, 0) \sim(\phi(x), 1)\} \cup_{\partial} D_{0}^{3}$.

(a) There exists a degree 1 map $h: M(\phi) \rightarrow D^{3}$ which is the identity on $D_{0}^{3}$.

(b) $H_{1}(M(\phi)) \cong \operatorname{coker}\left(\phi_{*}-\right.$ id: $\left.H_{1}(F) \rightarrow H_{1}(F)\right)$.

(c) If $p: \widetilde{M(\phi)} \rightarrow M(\phi)$ is the covering induced from $q: S^{3} \rightarrow D^{3}$ by $h: M(\phi) \rightarrow D^{3}$ then $H_{1}(\widetilde{M(\phi)}) \cong 60 \operatorname{coker}\left(\phi_{*}^{2}-\mathrm{id}: H_{1}(F) \rightarrow H_{1}(F)\right)$.

(d) $M(\phi), \widetilde{M(\phi)}$ are both irreducible and both contain incompressible tori.

Corollary (5.3). If $\phi_{*}^{2}-\mathrm{id}: H_{1}(F) \rightarrow H_{1}(F)$ is invertible then $M(\phi), \widetilde{M(\phi)}$ are homology 3-spheres and $p: \widetilde{M(\phi)} \rightarrow M(\phi)$ is the regular covering induced from $q: S^{3} \rightarrow D^{3}$ by means of the degree 1 map $h: M(\phi) \rightarrow D^{3}$.

Question. Is there a homology 3-sphere $M$ (with $\pi_{1}(M)$ infinite) that is not sufficiently large and such that there is a degree 1 map $h: M \rightarrow D^{3}$ with the corresponding regular covering $\widetilde{M}$ not a homology 3 -sphere?

We conclude with some examples.

Example. If $A=\left[\begin{array}{ll}a & b \\ c & d\end{array}\right]$ is an invertible $2 \times 2$ matrix over the integers such that $A^{2}-I$ is invertible (over the integers) then $\operatorname{det} A=-1$ and trace $A= \pm 1$. Conversely, if $A$ has determinant -1 and trace \pm 1 then $A, A-I$ and $A^{2}-I$ will all be invertible over the integers. It follows that there are no orientation preserving isomorphisms $\phi: S^{1} \times S^{1} \rightarrow S^{1} \times S^{1}$ such that $M(\phi)$ and $\widetilde{M(\phi)}$ are homology 3-spheres (see (5.3)).

Example. Suppose $A=\left[\begin{array}{ll}a & b \\ c & d\end{array}\right]$ has determinant 1. Then $A-I$ is invertible (over the integers) if, and only if, trace $A=1$ or 3 . If $A$ is any such matrix and $\phi: S^{1} \times S^{1} \rightarrow S^{1} \times S^{1}$ is the corresponding orientation preserving isomorphism then $M(\phi)$ is a homology 3-sphere, but $\widetilde{M(\phi)}$ will not be a homology 3-sphere. In fact, $H_{1}(\widetilde{M(\phi)}) \cong 60 \operatorname{coker}\left(A^{2}-I\right) \cong 60 Z_{3}$ (resp. 60Z 5 ) since 
$\operatorname{det}\left(A^{2}-I\right)=3($ resp. -5$)$ if $\operatorname{trace} A=1$ (resp. 3). As a particular example consider $A=\left[\begin{array}{cc}0 & -1 \\ 1 & 1\end{array}\right]$. Then

$$
A^{2}=\left[\begin{array}{rr}
-1 & -1 \\
1 & 0
\end{array}\right]=\left[\begin{array}{rr}
0 & 1 \\
-1 & 0
\end{array}\right]\left[\begin{array}{ll}
0 & -1 \\
1 & -1
\end{array}\right]\left[\begin{array}{rr}
0 & 1 \\
-1 & 0
\end{array}\right]^{-1}
$$

and therefore $M_{\phi}, M_{\phi^{2}}$ are orientable spherical space forms $\left(M_{5}, M_{3}\right.$ resp. in the notation of [LS]). $M(\phi)$ is a homology 3-sphere, but $H_{1}(\widetilde{M(\phi)}) \cong 60 Z_{3}$. Example. A matrix of the form $A=\left[\begin{array}{cc}P & I \\ -I & 0\end{array}\right]$, where all blocks are $g \times g$, is symplectic if, and only if, $P=P^{T}$. We have

$$
\operatorname{det}(A-\lambda I)=(-1)^{g} \operatorname{det}\left(\lambda P-\left(\lambda^{2}+1\right) I\right)
$$

and so $A \pm I$ will be invertible over the integers if, and only if, $\operatorname{det}(P+2 I)= \pm 1$ and $\operatorname{det}(P-2 I)= \pm 1$. If $g=2$ one can show that $P$ must have the form $P=\left[\begin{array}{ll}x & y \\ y & -x\end{array}\right]$, where $x^{2}+y^{2}=5$, i.e., $(x, y)$ must be one of $\pm(1,2), \pm(1,-2)$, $\pm(2,1), \pm(2,-1)$. A particular example when $g=3$ is given by

$$
P=\left[\begin{array}{rrr}
-2 & 1 & 1 \\
1 & -1 & 2 \\
1 & 2 & 0
\end{array}\right] \text {. }
$$

By taking direct sums of copies of these matrices for $g=2$ and $g=3$, we can find $g \times g$ matrices $P$, any $g \geq 2$, so that $\operatorname{det}(P+2 I)= \pm 1$ and $\operatorname{det}(P-2 I)= \pm 1$. It follows that the $2 g \times 2 g$ matrix $A=\left[\begin{array}{cc}P & I \\ -I & 0\end{array}\right]$ will be symplectic and satisfy $\operatorname{det}(A+I)= \pm 1, \operatorname{det}(A-I)= \pm 1$. Therefore, if $F$ is a closed orientable surface of genus $g \geq 2$ there are orientation preserving isomorphisms $\phi: F \rightarrow F$ so that $\phi_{*} \pm \mathrm{id}: H_{1}(F) \rightarrow H_{1}(F)$ are isomorphisms. According to (5.2) this means that $M(\phi), \widehat{M(\phi)}$ are homology 3-spheres.

\section{REFERENCES}

[D] M. N. Dyer, Homotopy classification of $(\pi, m)$-complexes, J. Pure Appl. Algebra 7 (1976), 249-282.

[Ha] J. Harer, Representing elements of $\pi_{1}\left(M^{3}\right)$ by fibered knots, Math. Proc. Cambridge Philos. Soc. 92 (1982), 133-138.

[He] J. Hempel, 3-manifolds, Ann. of Math. Studies, no. 86, Princeton Univ. Press, 1976.

[LS] E. Luft and D. Sjerve, 3-manifolds with subgroups $Z \oplus Z \oplus Z$ in their fundamental groups, Pacific J. Math. 114 (1984), 191-205.

[MSY] W. Meeks III, L. Simon and S. T. Yau, Embedded minimal surfaces, exotic spheres, and manifolds with positive Ricci curvature, Ann. of Math. (2) 116 (1982), 621-653.

[Ol] P. Olum, Mappings of manifolds and the notion of degree, Ann. of Math. (2) 58 (1953), 458480.

[O] P. Orlik, Seifert manifolds, Lecture Notes in Math., vol. 291, Springer-Verlag, Berlin and New York, 1972.

[PI] S. Plotnick, Homotopy equivalences and free modules, Topology 21 (1982), 91-99.

[Ro] D. Rolfsen, Knots and links, Math. Lecture Series, no. 7, Publish or Perish, 1976.

[S] H. Seifert, Topologie dreidimensionaler gefaserter Raüme, Acta Math. 60 (1933), 147-238; English translation in M. Seifert and W. Threlfall, A Textbook of Topology, Academic Press, 1980. 
[Sj] D. Sjerve, Homology spheres which are covered by spheres, J. London Math. Soc. (2) 6 (1973), 333-336.

[Sw 1 ] R. G. Swan, Induced representations and projective modules, Ann. of Math. (2) 71 (1960), 552-578.

[Sw 2 ] _, Projective modules over binary polyhedral groups, J. Reine Angew. Math. 342 (1983), 66-172.

Department of Mathematics, University of British Columbia, Vancouver, British Columbia, Canada, V6T 1Y4 